\title{
Rotational spectra of mono-substituted asymmetric $\mathrm{C}_{6} \mathrm{H}_{6}-\mathrm{H}_{2} \mathrm{O}$ dimers
}

\author{
B. Ram Prasad ${ }^{\text {a }}$, Mangala Sunder Krishnan ${ }^{\mathrm{a}, *}$, E. Arunan ${ }^{\mathrm{b}, *}$ \\ a Department of Chemistry, Indian Institute of Technology Madras, Chennai 600 036, India \\ ${ }^{\mathrm{b}}$ Department of Inorganic and Physical Chemistry, Indian Institute of Science, Bangalore 560 012, India
}

\begin{abstract}
This paper reports the assignment of the rotational spectra of the $m=0$ and 1 states of ${ }^{13} \mathrm{CC}_{5} \mathrm{H}_{6}-\mathrm{H}_{2} \mathrm{O}$ and $\mathrm{C}_{6} \mathrm{H}_{5} \mathrm{D}-\mathrm{H}_{2} \mathrm{O}$ dimers. The $m=1$ progression was not identified or assigned for both ${ }^{13} \mathrm{CC}_{5} \mathrm{H}_{6}-\mathrm{H}_{2} \mathrm{O}$ and $\mathrm{C}_{6} \mathrm{H}_{5} \mathrm{D}-\mathrm{H}_{2} \mathrm{O}$ in the earlier work, though for the symmetric isotopomers $\left(\mathrm{C}_{6} \mathrm{H}_{6}-\mathrm{H}_{2} \mathrm{O} / \mathrm{D}_{2} \mathrm{O} / \mathrm{H}_{2}{ }^{18} \mathrm{O}\right)$, they were identified [H.S. Gutowsky, T. Emilsson, E. Arunan, J. Chem. Phys. 99 (1993) 4883]. The $m=1$ transitions for ${ }^{13} \mathrm{CC}_{5} \mathrm{H}_{6}-\mathrm{H}_{2} \mathrm{O}$ and $\mathrm{C}_{6} \mathrm{H}_{5} \mathrm{D}-\mathrm{H}_{2} \mathrm{O}$ were split into two, unlike that of the parent $\mathrm{C}_{6} \mathrm{H}_{6}-\mathrm{H}_{2} \mathrm{O}$ isotopomer. The splitting varied, somewhat randomly, with quantum numbers $J$ and $K$. The $m=0$ lines of ${ }^{13} \mathrm{CC}_{5} \mathrm{H}_{6}-\mathrm{H}_{2} \mathrm{O}$ had significant overlap with the $m=1$ lines of the parent isotopomer, clouding proper assignment, and leading to an rms deviation of about $200 \mathrm{kHz}$ in the earlier work. The general semi-rigid molecular Hamiltonian coupled to an internal rotor, described recently by Duan et al. [Y.B. Duan, H.M. Zhang, K. Takagi, J. Chem. Phys. 104 (1996) 3914], is used in this work to assign both $m=0$ and 1 states of ${ }^{13} \mathrm{CC}_{5} \mathrm{H}_{6}-\mathrm{H}_{2} \mathrm{O}$ and $\mathrm{C}_{6} \mathrm{H}_{5} \mathrm{D}-\mathrm{H}_{2} \mathrm{O}$ dimers. Consequently, the $m=0$ fits for ${ }^{13} \mathrm{CC}_{5} \mathrm{H}_{6}-\mathrm{H}_{2} \mathrm{O} / \mathrm{D}_{2} \mathrm{O}$ have an rms deviation of only 4/ $7 \mathrm{kHz}$, comparable to experimental uncertainties. The fits for $m=1$ transitions for ${ }^{13} \mathrm{CC}_{5} \mathrm{H}_{6}-\mathrm{H}_{2} \mathrm{O}$ and $\mathrm{C}_{6} \mathrm{H}_{5} \mathrm{D}-\mathrm{H}_{2} \mathrm{O}$ dimers have an rms deviation of about $200 \mathrm{kHz}$. However, it is of the same order of magnitude as that of the $m=1$ state of the parent $\mathrm{C}_{6} \mathrm{H}_{6}-\mathrm{H}_{2} \mathrm{O}$ dimer. The $A$ rotational constants determined from the $m=0$ fits for both ${ }^{13} \mathrm{CC}_{5} \mathrm{H}_{6}-\mathrm{H}_{2} \mathrm{O}$ and ${ }^{13} \mathrm{CC}_{5} \mathrm{H}_{6}-\mathrm{D}_{2} \mathrm{O}$ isotopomers are identical and very close to the $C$ rotational constant for ${ }^{13} \mathrm{CC}_{5} \mathrm{H}_{6}$. This provides a direct experimental determination for the $C$ rotational constant of ${ }^{13} \mathrm{CC}_{5} \mathrm{H}_{6}$, which has a negligible dipole moment.
\end{abstract}

\section{Introduction}

The $\mathrm{C}_{6} \mathrm{H}_{6}-\mathrm{H}_{2} \mathrm{O}$ complex has attracted significant attention as a prototype for aromatic-polar interactions [1-6]. Due to the large amplitude motion of the $\mathrm{H}_{2} \mathrm{O}$, within the complex, it has a complicated rotational spectrum which is yet to be fully understood. Blake and coworkers [5] reported the first observation of the $J=4-10$ rotational spectra in which they had unambiguously assigned the ground state symmetric-top spectrum. Two other unassigned progressions were reported. Shortly thereafter, Gutowsky et al. [6]

\footnotetext{
* Corresponding authors. Fax: +91 8023601552 (E. Arunan).

E-mail addresses: mangal@iitm.ac.in (M.S. Krishnan), arunan@ ipc.iisc.ernet.in (E. Arunan).
}

reported the observation of $J=0-4$ transitions in which they confirmed and refined the ground state symmetrictop spectrum. Also, they noted that there was one strong line between the two unassigned progressions. The line in the middle and the two progressions appeared as one long progression. The newer line was assigned as a $K=0$ line of the internal rotor progression $(m=1)$ using the following expression:

$$
\begin{aligned}
v= & 2(J+1)\left\{B-D_{J K} K^{2}-D_{J m} m^{2}-D_{J K m} K m\right. \\
& \left.-H_{J K m} K^{2} m^{2}\right\}-4(J+1)^{3} D_{J} .
\end{aligned}
$$

This expression had been used earlier by Fraser et al. [7] for assigning the spectrum of the symmetric-top complex, $\mathrm{CF}_{3} \mathrm{H}-\mathrm{NH}_{3}$. Here, $m$ is the projection of the internal rotational angular momentum on the principal axis. 
This is basically the standard symmetric-top equation [8] modified for an internal rotor on the symmetry axis. One minor modification required for the $\mathrm{C}_{6} \mathrm{H}_{6}-\mathrm{H}_{2} \mathrm{O}$ spectrum was the addition of the $-D_{J J m} m^{2}$ to the $D_{J}$ term [6]. Eq. (1) leads to a simple symmetric-top spectrum for the $m=0$ ground state as observed. For $m=1$, it predicts $2 J+1$ lines corresponding to $m K=-J$ to $+J$. Since Blake and coworkers used a Stark modulated spectrometer, the $K=0$ line at the center of this progression could not be identified in their work. Though Eq. (1) could explain the broad picture of $\mathrm{C}_{6} \mathrm{H}_{6}-\mathrm{H}_{2} \mathrm{O}$ rotational spectrum, there were some anomalies that remain unresolved. The major anomaly is that the $m K=+J$ line was missing and an odd line appeared above the $m=0$ progression; see Fig. 1 . Similar pattern was found in the rotational spectra of $\mathrm{Rg}-\mathrm{C}_{6} \mathrm{H}_{6}-\mathrm{H}_{2} \mathrm{O}$ sandwich trimers $(\mathrm{Rg}=\mathrm{Ar}, \mathrm{Ne}$ or $\mathrm{Kr})$ $[9,10]$, suggesting that it was not an isolated phenomenon.

The spectra for the mono-substituted dimers containing ${ }^{13} \mathrm{CC}_{5} \mathrm{H}_{6}$ and $\mathrm{C}_{6} \mathrm{H}_{5} \mathrm{D}[6]$ were more complicated. The rms deviation for the $m=0$ lines of ${ }^{13} \mathrm{CC}_{5} \mathrm{H}_{6}$ $\mathrm{H}_{2} \mathrm{O} / \mathrm{D}_{2} \mathrm{O}$ was more than $200 \mathrm{kHz}$. For the $m=1$ progressions of ${ }^{13} \mathrm{CC}_{5} \mathrm{H}_{6}-\mathrm{H}_{2} \mathrm{O}$ and $\mathrm{C}_{6} \mathrm{H}_{5} \mathrm{D}-\mathrm{H}_{2} \mathrm{O}$, only the $K=0$ lines of $J=0 \rightarrow 1$ could be identified. These were all split into a doublet with different splittings for different isotopomers (Table 7 in [6]). The higher $J$ lines could not be identified or assigned. The larger rms deviation observed in the fit of the $m=0$ lines was due to the fact that the $m=1$ lines of the parent $\mathrm{C}_{6} \mathrm{H}_{6}-\mathrm{H}_{2} \mathrm{O}$ and $m=0$ lines of ${ }^{13} \mathrm{CC}_{5} \mathrm{H}_{6}-\mathrm{H}_{2} \mathrm{O}$ were overlapping. The splitting observed in the $J=0 \rightarrow 1, m K=0$ line could be reproduced with an effective twofold barrier using the ITRFIT code by Taleb-Bendiab et al. [11] based on the principal axis method (PAM) Hamiltonian. This Hamiltonian is a truncated form of the Hamiltonian used by Rohart [12]. However, such a fit resulted in the prediction of higher $J$ lines with similar splittings and these were not found. For the higher $J$ lines, the splitting showed a non-trivial dependence on $J$ and $K$ for the nearly prolate asymmetric-top complexes. Attempts were made to fit the spectra initially using the full Hamiltonian of Rohart but the fit was not satisfactory. Several other Hamiltonian forms for an analysis of torsion have been described elsewhere [13-16].

In a series of papers, Duan et al. $[17,18]$ have employed the van Vleck transformation method to study the vibration-rotation-torsion (VRT) Hamiltonian. All the terms described by Rohart [12] and Taleb-Bendiab et al. [11] are part of the more general, systematic scheme of derivation given by Duan et al. The $D_{J J m}$ term used in an adhoc manner in our earlier work on $\mathrm{C}_{6} \mathrm{H}_{6}-\mathrm{H}_{2} \mathrm{O}[6]$ is equivalent to the $g_{v}$ term in Duan et al.'s work [18]. Their derivation is based on the Watson Hamiltonian [19]. By introducing a slightly different ordering scheme, they have derived expressions for an effective VRT Hamiltonian and an effective torsional-rotational Hamiltonian for a given vibrational state. They have also examined the coupling of rotation to torsion. In our approach to be presented below, we have used their formalism and have proposed an effective torsional-rotational Hamiltonian to analyze the spectra of benzene-water dimers. We have used the ITRFIT FORTRAN code provided by Taleb-Bendiab [11] with appropriate modifications to include all the terms described in [17] and [18]. The application of this Hamiltonian to the analysis of spectra of van der Waals complexes is done for the first time to our knowledge. With this Hamiltonian, both the $m=0$ and 1 progressions for ${ }^{13} \mathrm{CC}_{5} \mathrm{H}_{6}-\mathrm{H}_{2} \mathrm{O}$ and $\mathrm{C}_{6} \mathrm{H}_{5} \mathrm{D}-\mathrm{H}_{2} \mathrm{O}$ could be assigned and fitted to the same degree of accuracy as for the parent isotopomer. The results are presented below.

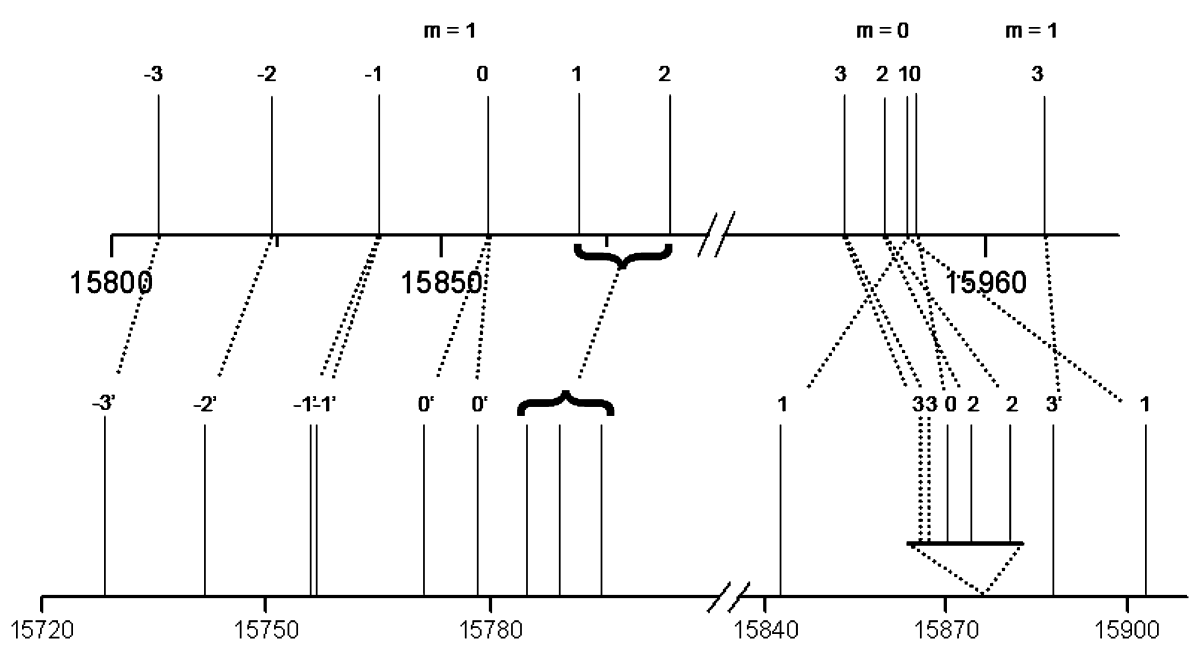

Fig. 1. Stick diagram of the observed $J=3 \rightarrow 4$ spectrum for $\mathrm{C}_{6} \mathrm{H}_{6}-\mathrm{H}_{2} \mathrm{O}$ (top) and ${ }^{13} \mathrm{CC}_{5} \mathrm{H}_{6}-\mathrm{H}_{2} \mathrm{O}$ (bottom) complexes. For ${ }^{13} \mathrm{CC}_{5} \mathrm{H}_{6}-\mathrm{H}_{2} \mathrm{O}$, the $K=3$ lines in the $m=0$ progression are shown as dashed lines as these are from predictions. They are very close to the strong $m K=1$ line of $\mathrm{C}_{6} \mathrm{H}_{6}-\mathrm{H}_{2} \mathrm{O}$. 
The experimental spectra were obtained with the Balle-Flygare spectrometer described elsewhere [6]. The details of the spectrometer and its applications have been summarized in a recent review [20]. The ${ }^{13} \mathrm{CC}_{5} \mathrm{H}_{6}-\mathrm{H}_{2} \mathrm{O}$ lines could be unambiguously assigned using the ${ }^{13} \mathrm{C}$ depleted benzene and it turned out to be quite useful in the assignment.

\section{Results and discussion}

The $m=0$ and 1 transitions for $\mathrm{C}_{6} \mathrm{H}_{6}-\mathrm{H}_{2} \mathrm{O}$ and ${ }^{13} \mathrm{CC}_{5} \mathrm{H}_{6}-\mathrm{H}_{2} \mathrm{O}$ are shown as a stick diagram in Fig. 1. The similarity in the $m=1$ progression for the two complexes is evident. The transition frequencies for the two complexes were analyzed using an equilibrium structure, in which $\mathrm{H}_{2} \mathrm{O}$ is the rotor and the substituted benzene is the frame. These frequencies are given in Tables 1 $(m=0)$ and $3(m=1)$ and the fitting parameters are given in Tables 2 and 4, respectively. From Fig. 1 and Table 3 , it can be noted that every transition in parent isotopomer is split into two in the mono-substituted benzene, and the splitting varies with $J$ and $K$. The details of the fitting are given below.

The $m=0$ transitions were fitted earlier [6] for the near-prolate asymmetric tops ${ }^{13} \mathrm{CC}_{5} \mathrm{H}_{6}-\mathrm{H}_{2} \mathrm{O} / \mathrm{D}_{2} \mathrm{O}$ and $\mathrm{C}_{6} \mathrm{H}_{5} \mathrm{D}-\mathrm{H}_{2} \mathrm{O} / \mathrm{D}_{2} \mathrm{O}$, using the basic treatment of Kivelson and Wilson [14] by including only two centrifugal distortion constants, namely $D_{J}$ and $D_{J K}$. The residues were within experimental uncertainty in the case of $\mathrm{C}_{6} \mathrm{H}_{5} \mathrm{D}-\mathrm{H}_{2} \mathrm{O}$ and $\mathrm{C}_{6} \mathrm{H}_{5} \mathrm{D}-\mathrm{D}_{2} \mathrm{O}$. The rms deviations

Table 1

The revised assignment of rotational transition frequencies for nearly prolate asymmetric-top isotopomers ${ }^{13} \mathrm{CC}_{5} \mathrm{H}_{6}-\mathrm{H}_{2} \mathrm{O}$ and ${ }^{13} \mathrm{CC}_{5} \mathrm{H}_{6}-\mathrm{D}_{2} \mathrm{O}$ for the $m=0$ ground state ${ }^{\mathrm{a}}$

\begin{tabular}{|c|c|c|c|c|}
\hline \multirow{2}{*}{$\begin{array}{l}\text { Transition } \\
J_{p o}^{\prime \prime} \rightarrow J_{p o}^{\prime}\end{array}$} & \multicolumn{2}{|c|}{${ }^{13} \mathrm{CC}_{5} \mathrm{H}_{6}-\mathrm{H}_{2} \mathrm{O}$} & \multicolumn{2}{|c|}{${ }^{13} \mathrm{CC}_{5} \mathrm{H}_{6}-\mathrm{D}_{2} \mathrm{O}$} \\
\hline & Obs (MHz) & Res (MHz) & Obs (MHz) & Res (MHz) \\
\hline $0_{00} \rightarrow 1_{00}$ & 3968.560 & 0.004 & 3803.896 & 0.009 \\
\hline $1_{11} \rightarrow 2_{12}$ & 7921.737 & -0.002 & 7593.557 & -0.017 \\
\hline $1_{01} \rightarrow 2_{02}$ & 7936.828 & -0.001 & 7607.540 & 0.009 \\
\hline $1_{10} \rightarrow 2_{11}$ & 7952.030 & 0.001 & 7621.434 & -0.002 \\
\hline $2_{12} \rightarrow 3_{13}$ & 11882.279 & -0.003 & 11390.045 & -0.001 \\
\hline $2_{02} \rightarrow 3_{03}$ & 11904.535 & 0.000 & 11410.687 & -0.001 \\
\hline $2_{21} \rightarrow 3_{22}$ & 11904.470 & 0.007 & 11410.228 & 0.007 \\
\hline $2_{20} \rightarrow 3_{21}$ & - & b & 11410.849 & 0.002 \\
\hline $2_{11} \rightarrow 3_{12}$ & 11927.710 & -0.005 & 11431.837 & -0.002 \\
\hline $3_{13} \rightarrow 4_{14}$ & 15842.437 & 0.002 & 15186.148 & 0.004 \\
\hline $3_{03} \rightarrow 4_{04}$ & 15871.393 & 0.001 & 15213.118 & 0.003 \\
\hline $3_{22} \rightarrow 4_{23}$ & 15872.078 & -0.006 & 15213.093 & -0.008 \\
\hline $3_{31} \rightarrow 4_{32}$ & - & $\mathrm{b}$ & 15211.710 & -0.001 \\
\hline $3_{30} \rightarrow 4_{31}$ & - & $\mathrm{b}$ & 15211.719 & 0.002 \\
\hline $3_{21} \rightarrow 4_{22}$ & 15874.112 & 0.001 & 15214.668 & 0.002 \\
\hline $3_{12} \rightarrow 4_{13}$ & 15903.007 & 0.003 & 15241.860 & -0.002 \\
\hline
\end{tabular}

${ }^{\text {a }}$ Revised from Table 5 of [6].

${ }^{b}$ Not included in the fit. These lines have significant overlap with the much stronger $m=1$ lines of the parent at 11905.11 and 15870.97 (see Table 3).
Table 2

Rotational constants determined from the $m=0$ transitions of the nearly prolate asymmetric-top isotopomers ${ }^{13} \mathrm{CC}_{5} \mathrm{H}_{6}-\mathrm{H}_{2} \mathrm{O}$ and ${ }^{13} \mathrm{CC}_{5} \mathrm{H}_{6}-\mathrm{D}_{2} \mathrm{O}$

\begin{tabular}{lll}
\hline Constant $^{\mathrm{a}}$ & ${ }^{13} \mathrm{CC}_{5} \mathrm{H}_{6}-\mathrm{H}_{2} \mathrm{O}$ & ${ }^{13} \mathrm{CC}_{5} \mathrm{H}_{6}-\mathrm{D}_{2} \mathrm{O}$ \\
\hline$A(\mathrm{MHz})$ & $2832(2)$ & $2832(3)$ \\
$B(\mathrm{MHz})$ & $1991.857(8)$ & $1908.916(1)$ \\
$C(\mathrm{MHz})$ & $1976.712(8)$ & $1894.985(1)$ \\
$D_{J}(\mathrm{kHz})$ & $3.34(2)$ & $3.61(3)$ \\
$D_{J K}(\mathrm{kHz})$ & $36.2(1)$ & $45.5(1)$ \\
$\sigma(\mathrm{kHz})$ & 4.4 & 7.5 \\
\hline \hline
\end{tabular}

${ }^{\text {a }}$ The numbers in parentheses represent standard deviation.

were significantly higher $(>200 \mathrm{kHz})$ for ${ }^{13} \mathrm{CC}_{5} \mathrm{H}_{6}-\mathrm{H}_{2} \mathrm{O}$ and ${ }^{13} \mathrm{CC}_{5} \mathrm{H}_{6}-\mathrm{D}_{2} \mathrm{O}$ [6]. This was due to the overlap of $m=1$ lines of the parent isotopomer, leading to the wrong assignment of ${ }^{13} \mathrm{CC}_{5} \mathrm{H}_{6}-\mathrm{H}_{2} \mathrm{O}$ and ${ }^{13} \mathrm{CC}_{5} \mathrm{H}_{6}$ $\mathrm{D}_{2} \mathrm{O}$ transitions earlier. The corrected assignments for transitions with $m=0$ for ${ }^{13} \mathrm{CC}_{5} \mathrm{H}_{6}-\mathrm{H}_{2} \mathrm{O}$ and ${ }^{13} \mathrm{CC}_{5} \mathrm{H}_{6}-\mathrm{D}_{2} \mathrm{O}$ are given in Table 1 . The spectroscopic constants are given in Table 2. The errors are within the experimental uncertainty in both cases. The $2_{20} \rightarrow 3_{21}$ and $3_{30} \rightarrow 4_{31} / 3_{31} \rightarrow 4_{32}$ (both being degenerate) transitions of ${ }^{13} \mathrm{CC}_{5} \mathrm{H}_{6}-\mathrm{H}_{2} \mathrm{O}$ were very close to the much stronger $\mathrm{mK}=1$ transitions of $\mathrm{C}_{6} \mathrm{H}_{6}-\mathrm{H}_{2} \mathrm{O}$ and could not be identified independently. It may be noticed that the $A$ rotational constant of ${ }^{13} \mathrm{CC}_{5} \mathrm{H}_{6}-\mathrm{H}_{2} \mathrm{O}$ and ${ }^{13} \mathrm{CC}_{5} \mathrm{H}_{6}-\mathrm{D}_{2} \mathrm{O}$ are both identical at $2832 \mathrm{MHz}$. It is very close to the $C$ rotational constant of ${ }^{13} \mathrm{CC}_{5} \mathrm{H}_{6}$ (2813 MHz) calculated from its structure. As ${ }^{13} \mathrm{CC}_{5} \mathrm{H}_{6}$ has a negligible dipole moment, an experimental determination of its rotational constant is difficult, if not impossible. Rotational spectrum of ${ }^{13} \mathrm{CC}_{5} \mathrm{H}_{6}$ has not been observed yet. Rotationally resolved vibrational spectra of ${ }^{13} \mathrm{CC}_{5} \mathrm{H}_{6}$ have been reported [21,22]. However, in these reports, the rotational constants of ${ }^{13} \mathrm{CC}_{5} \mathrm{H}_{6}$ have not been independently determined but calculated from the structure of $\mathrm{C}_{6} \mathrm{H}_{6}$. On the other hand, $\mathrm{C}_{6} \mathrm{H}_{5} \mathrm{D}$ has a small observable dipole moment and its rotational spectrum has been observed [23]. It has a $C$ constant $(2749.7 \mathrm{MHz})$ which is also close to the $A$ rotational constant $(2765.5 \mathrm{MHz})$ of the corresponding water dimers, $\mathrm{C}_{6} \mathrm{H}_{5} \mathrm{D}-\mathrm{H}_{2} \mathrm{O} / \mathrm{C}_{6} \mathrm{H}_{5} \mathrm{D}-\mathrm{D}_{2} \mathrm{O}$ $[6,20]$. Thus, a direct determination of $C$ rotational constants of mono-substituted benzene isotopomers is possible from the data given here. In these complexes, water acts as an electrophore [20] for the determination of rotational constants of ${ }^{13} \mathrm{CC}_{5} \mathrm{H}_{6}$ and $\mathrm{C}_{6} \mathrm{H}_{5} \mathrm{D}$.

As mentioned earlier, the $m=1$ progression for ${ }^{13} \mathrm{CC}_{5} \mathrm{H}_{6}-\mathrm{H}_{2} \mathrm{O}$ and $\mathrm{C}_{6} \mathrm{H}_{5} \mathrm{D}-\mathrm{H}_{2} \mathrm{O}$ could not be assigned at all in the earlier work. However, an interesting regularity was observed in the $\mathbf{J}=0 \rightarrow 1$ transitions of the $m=1$ progression for the two isotopomers. They form a doublet, with a splitting of $0.3-1.3 \mathrm{MHz}$ for various isotopomers. For the two isotopomers noted above, the doublets appear at frequencies which are 25.9 and 
Table 3

Observed rotational transition frequencies and residues from the fit (in $\mathrm{MHz}$ ) for benzene-water isotopomers in $m=1$ excited internal rotor state

\begin{tabular}{|c|c|c|c|c|c|c|c|}
\hline \multirow{2}{*}{$\begin{array}{l}\text { Transition } \\
J^{\prime \prime} \rightarrow J^{\prime}\end{array}$} & \multirow[t]{2}{*}{$K^{\mathrm{a}}$} & \multicolumn{2}{|l|}{$\mathrm{C}_{6} \mathrm{H}_{6}-\mathrm{H}_{2} \mathrm{O}$} & \multicolumn{2}{|l|}{$\mathrm{C}_{6} \mathrm{H}_{5} \mathrm{D}-\mathrm{H}_{2} \mathrm{O}$} & \multicolumn{2}{|c|}{${ }^{13} \mathrm{CC}_{5} \mathrm{H}_{6}-\mathrm{H}_{2} \mathrm{O}$} \\
\hline & & Obs $(\mathrm{MHz})$ & Res (MHz) & Obs $(\mathrm{MHz})$ & Res (MHz) & Obs (MHz) & Res (MHz) \\
\hline $0 \rightarrow 1$ & 0 & 3963.2947 & -0.503 & $\begin{array}{l}3911.5460 \\
3912.4989\end{array}$ & $\begin{array}{r}0.008 \\
-0.153\end{array}$ & $\begin{array}{l}3942.4820 \\
3942.9000\end{array}$ & $\begin{array}{l}0.001 \\
0.000\end{array}$ \\
\hline $1 \rightarrow 2$ & $\begin{array}{r}-1 \\
0\end{array}$ & $\begin{array}{r}7919.7245 \\
26.9889 \\
81.4323\end{array}$ & $\begin{array}{l}-0.108 \\
0.369 \\
\text { c }\end{array}$ & $\begin{array}{r}7806.6607 \\
07.6401 \\
22.8458 \\
26.0790 \\
73.6050 \\
73.2777\end{array}$ & $\begin{array}{r}-0.466 \\
0.458 \\
0.025 \\
-0.120 \\
\mathrm{c} \\
\mathrm{c}\end{array}$ & $\begin{array}{r}7878.3400 \\
78.8600 \\
85.0904 \\
86.4805 \\
939.4500 \\
939.4500\end{array}$ & $\begin{array}{r}-0.256 \\
0.256 \\
0.001 \\
-0.002 \\
\mathrm{c} \\
\mathrm{c}\end{array}$ \\
\hline $2 \rightarrow 3$ & $\begin{array}{r}-2 \\
-1 \\
0\end{array}$ & $\begin{array}{r}11868.1338 \\
79.9854 \\
91.4670 \\
905.1077 \\
71.9417\end{array}$ & $\begin{array}{r}0.092 \\
-0.081 \\
0.050 \\
\text { b }\end{array}$ & $\begin{array}{r}11690.3032 \\
690.3032 \\
715.4804 \\
15.8555 \\
33.5977 \\
41.7748 \\
54.0581 \\
59.8707 \\
809.1890 \\
10.1433\end{array}$ & $\begin{array}{r}0.023 \\
0.018 \\
-0.099 \\
0.016 \\
0.012 \\
0.252 \\
\mathrm{~b} \\
\mathrm{~b} \\
\mathrm{c} \\
\mathrm{c}\end{array}$ & $\begin{array}{r}11806.5800 \\
806.5800 \\
17.9680 \\
17.9680 \\
27.9570 \\
31.4173 \\
43.2430 \\
45.5760 \\
909.0557 \\
909.0557\end{array}$ & $\begin{array}{r}0.000 \\
0.000 \\
0.019 \\
-0.018 \\
0.007 \\
-0.007 \\
\mathrm{~b} \\
\mathrm{~b} \\
\mathrm{c} \\
\mathrm{c}\end{array}$ \\
\hline $3 \rightarrow 4$ & $\begin{array}{r}-3 \\
-2 \\
-1 \\
0\end{array}$ & $\begin{array}{r}15807.3407 \\
24.5635 \\
40.7324 \\
57.1318\end{array}$ & $\begin{array}{r}-0.019 \\
0.083 \\
-0.269 \\
0.341 \\
\\
-0.231 \\
0.046\end{array}$ & $\begin{array}{r}15552.3150 \\
52.3150 \\
89.0712 \\
89.0712 \\
621.2891 \\
22.2396 \\
43.5432 \\
59.3721 \\
15660.6510 \\
81.4077 \\
95.5970 \\
15735.1465 \\
35.1465 \\
\end{array}$ & $\begin{array}{l}0.004 \\
0.001 \\
-0.02 \\
-0.053 \\
0.025 \\
0.102 \\
-0.025 \\
-0.113 \\
\mathrm{~b} \\
\mathrm{~b} \\
\mathrm{~b} \\
\mathrm{c} \\
\mathrm{c} \\
\end{array}$ & $\begin{array}{r}15725.5720 \\
725.5720 \\
42.0676 \\
42.0676 \\
58.0441 \\
59.1323 \\
71.2018 \\
78.4084 \\
15785.100 \\
89.3121 \\
94.8868 \\
15878.5023 \\
15878.5023 \\
\end{array}$ & $\begin{array}{r}-0.053 \\
-0.054 \\
0.001 \\
0.000 \\
-0.481 \\
0.479 \\
0.005 \\
-0.005 \\
\mathrm{~b} \\
\mathrm{~b} \\
\mathrm{~b} \\
\mathrm{c} \\
\mathrm{c}\end{array}$ \\
\hline
\end{tabular}

${ }^{\text {a }} K$ is not sufficient to specify the quantum state for the asymmetric-top isotopomers. The fitting used $\tau$ instead, where $\tau$ goes from 1 to $2 J+1 /$ $4 J+2$ for symmetric/asymmetric isotopomers with increasing energy. However, $K$ is retained in this table for simplicity following Eq. (1). See Table 5 .

${ }^{\mathrm{b}}$ Not included in the fit. For the $J=3 \rightarrow 4, K=1$ and 2, instead of 4 lines (two doublets), only three lines have been observed for both asymmetric isotopomers. It is possibly due to some local perturbation that has not been considered; see text and Fig. 1 for details. The deviations for these lines are a few MHz.

${ }^{\mathrm{c}}$ The $m k=J$ lines are missing from the $m=1$ progression. Another line has been observed above the $m=0, K=0$ line for all isotopomers and this has been included as a likely candidate for the missing line. See Fig. 1.

25.0 $\mathrm{MHz}$ lower than the corresponding transitions (singlet) of the $m=0$ state, respectively. For the parent isotopomer, this difference is $26.2 \mathrm{MHz}$. On this basis, the doublets have been assigned to the $m=1$ state of the asymmetric tops. It was noted that the splitting for $\mathrm{C}_{6} \mathrm{H}_{5} \mathrm{D}-\mathrm{H}_{2} \mathrm{O}$ is about twice that of ${ }^{13} \mathrm{CC}_{5} \mathrm{H}_{6}-\mathrm{H}_{2} \mathrm{O}$. Also, the splitting for each isotopomer changes systematically with isotopic substitution in water. The observed splitting is the result of the asymmetry arising due to substitution and the change in the effective potential for internal rotation of $\mathrm{H}_{2} \mathrm{O}$ in the complex. The ${ }^{13} \mathrm{C} / \mathrm{D}$ substitution introduces a small perturbation in the potential, which is represented by a $V_{2}$ term. Such effective $V_{1}$ and $V_{2}$ potential terms have been used in explaining the microwave spectrum of $\mathrm{CH}_{2} \mathrm{DOH}$ by Quade and
Suenram [24] earlier. A twofold effective barrier was essential for the splitting of $m=1$ energy levels removing the degeneracy due to positive and negative values of $m K$. However, the internal rotation of $\mathrm{H}_{2} \mathrm{O}$ does not lead to inversion of dipole moments and therefore selection rules for the observed ' $a$ ' dipole transitions connect lower $\leftrightarrow$ lower and upper $\leftrightarrow$ upper levels.

The assignments for the $m=1$ state of ${ }^{13} \mathrm{CC}_{5} \mathrm{H}_{6}-\mathrm{H}_{2} \mathrm{O}$ and $\mathrm{C}_{6} \mathrm{H}_{5} \mathrm{D}-\mathrm{H}_{2} \mathrm{O}$ are given in Table 3 and are reported for the first time. These two complexes are nearly prolate asymmetric tops. Several attempts were made earlier to identify and assign the transitions of the $m=1$ state for these asymmetric tops, but with only limited success. In the present paper, the transition frequencies have been fitted by including several higher order and 
Table 4

Spectroscopic constants for benzene-water isotopomers derived using the internal rotation Hamiltonian

\begin{tabular}{llll}
\hline Constant $^{\mathrm{a}}$ & $\mathrm{C}_{6} \mathrm{H}_{6}-\mathrm{H}_{2} \mathrm{O}$ & $\mathrm{C}_{6} \mathrm{H}_{5} \mathrm{D}-\mathrm{H}_{2} \mathrm{O}$ & ${ }^{13} \mathrm{CC}_{5} \mathrm{H}_{6}-\mathrm{H}_{2} \mathrm{O}$ \\
\hline$A(\mathrm{MHz})$ & - & $2787(1)$ & $2785(1)$ \\
$B(\mathrm{MHz})$ & $1994.77(4)$ & $1992.00(3)$ & $1991.86(3)$ \\
$C(\mathrm{MHz})$ & 1994.7735 (Fixed) & $1944.99(3)$ & $1976.71(3)$ \\
$D_{J m}(\mathrm{MHz})$ & $-13.12(4)$ & $-13.3(1)$ & $-13.3(1)$ \\
$L_{J a}(\mathrm{MHz})$ & $-1.77(2)$ & $-5.2(1)$ & $-1.72(9)$ \\
$D_{J}(\mathrm{kHz})$ & $3.3(9)$ & $3(1)$ & $3.1(9)$ \\
$D_{J K}(\mathrm{kHz})$ & $37(2)$ & $29(2)$ & $40(5)$ \\
$D_{J K m}(\mathrm{kHz})$ & $-34(6)$ & $139(74)$ & $183(61)$ \\
$D_{J J m}(\mathrm{kHz})$ & $17(1)$ & $-86(5)$ & $-26(4)$ \\
$l_{V}(\mathrm{kHz})$ & $0($ Fixed $)$ & $148(10)$ & $-26(8)$ \\
$O_{V}(\mathrm{kHz})$ & $0($ Fixed) & $457(71)$ & $163(60)$ \\
$o_{V}(\mathrm{kHz})$ & $0($ Fixed) & $48(2)$ & $23(2)$ \\
$h_{J 3}(\mathrm{kHz})$ & $0.18(9)$ & $-4.8(9)$ & $-1.8(7)$ \\
$h_{J 6}(\mathrm{kHz})$ & $-0.15(4)$ & $-4.1(3)$ & $0.5(2)$ \\
$V_{2}(\mathrm{MHz})$ & $0($ Fixed $)$ & $1.906($ Fixed $)$ & $0.839($ Fixed $)$ \\
$\sigma(\mathrm{MHz})$ & 0.18 & 0.21 & 0.17 \\
$\mathrm{~N}^{\mathrm{c}}$ & $22 / 26$ & $36 / 47$ & $33 / 44$ \\
\hline \hline
\end{tabular}

a The numbers in parentheses represent standard deviation.

b The fit is insensitive to the value of the barrier in this order of magnitude and has been fixed as twice the splitting observed in the $J=0 \rightarrow 1$ transition.

${ }^{\mathrm{c}}$ No. of transitions fitted/observed.

coupling terms to the general semi-rigid molecular Hamiltonian coupled to an internal rotor $[17,18]$. The Hamiltonian used to fit the spectra reported in this paper is

$$
\begin{aligned}
H= & A P_{a}^{2}+B P_{b}^{2}+C P_{c}^{2}-2 Q_{a} P_{a} p+F p^{2} \\
& +\frac{1}{2} V_{2}(1-\cos 2 \gamma)+H_{\mathrm{dist}},
\end{aligned}
$$

where

$$
\begin{aligned}
H_{\text {dist }}= & -D_{J} P^{4}-D_{J K} P^{2} P_{a}^{2}+D_{J m} P^{2} P^{2} \gamma+D_{J K m} P^{2} P_{a}^{2} P_{\gamma}^{2} \\
& +D_{J J m} P^{4} P_{\gamma}^{2}+L_{J a} P^{2} P_{a} P_{\gamma}+l_{v} P^{4} P_{a} P_{\gamma}+h_{J 6} P^{6} P_{a} P_{\gamma} \\
& +h_{J 3} P^{4} P_{a}^{3} P_{\gamma}+O_{v} P^{2}\left\{P_{\gamma}^{2},(1-\cos 2 \gamma)\right\} \\
& +o_{v} P^{4}\left\{P_{\gamma}^{2},(1-\cos 2 \gamma)\right\}
\end{aligned}
$$

and

$A=A_{r}+F \rho_{a}^{2}, \quad B=B_{r}, \quad C=C_{r}$,

$Q_{a}=F \rho_{a}, \quad F=F_{\gamma}\left[1-\rho_{a} \cos \theta_{a}\right]^{-1}$,

$\rho_{a}=\frac{A_{r}}{F_{\gamma}} \cos \theta_{a}$.

Here, $P_{a}, P_{b}$, and $P_{c}$ are the projections of the total angular momentum onto the principal axes in the $I^{r}$ representation; $p$ is the operator for angular momentum of the internal rotor; $\alpha$ is the internal rotation angle; $V_{2}$ is the effective barrier height; $A_{r}, B_{r}$, and $C_{r}$ are rigid rotor rotational constants in the principal axes; $F_{\gamma}$ is proportional to the inverse moment of inertia $I_{\gamma}$ of the internal rotor $\left(I_{b}\right.$ of $\left.\mathrm{H}_{2} \mathrm{O}\right)$; and $\theta_{a}$ is the angle between the internal rotor axis and the " $a$ " axis. In our model, the internal rotor axis coincides with the " $a$ " axis of the complex, i.e., $\theta_{a}$ is $0^{\circ}$. No improvements were observed in the fit when $\theta_{a}$ was varied. In $H_{\text {dist }}$ (Hamiltonian with distortion coefficients), the terms with coefficients $D_{J}$ and $D_{J K}$ represent the usual centrifugal distortions in the Watson's reduced Hamiltonian [25]. The remaining terms represent distortion contribution due to internal rotation and its coupling with the overall rotation. Note that $L_{J a}$ and $D_{J K m}$ in Eq. (2) are the same as $D_{J K m}$ and $H_{J K m}$ used in [6].

The Hamiltonian matrix was constructed using $|J K M m\rangle$ basis set, where $J, K$, and $M$ are quantum numbers associated with symmetric-top wave functions and $m$ is the quantum number associated with the free internal rotor wave functions. Since $m$ has no finite limits, the Hamiltonian matrix is infinite in principle. However, the Hamiltonian matrix was truncated at $m= \pm 2$ for ensuring convergence of lower energy levels.

For $\mathrm{C}_{6} \mathrm{H}_{5} \mathrm{D}-\mathrm{H}_{2} \mathrm{O}$ and ${ }^{13} \mathrm{CC}_{5} \mathrm{H}_{6}-\mathrm{H}_{2} \mathrm{O}$ the torsionalrotational transitions listed in Table 3 were fit using the internal rotation Hamiltonian given in Eq. (2), which includes the effects of centrifugal distortion and torsion-rotation couplings in the form of $H_{\text {dist }}$. The terms of $H_{\text {dist }}$ given below are additional to what was used earlier in the fit of parent compound [6]

$$
\begin{aligned}
& l_{v} P^{4} P_{a} P_{\gamma}+h_{J 6} P^{6} P_{a} P_{\gamma}+h_{J 3} P^{4} P_{a}^{3} P_{\gamma} \\
& \quad+O_{v} P^{2}\left\{P_{\gamma}^{2},(1-\cos 2 \gamma)\right\}+o_{v} P^{4}\left\{P_{\gamma}^{2},(1-\cos 2 \gamma)\right\} .
\end{aligned}
$$

The distortion parameters are the same as the parameters used by Duan et al. [17,18] in their analysis of $\mathrm{CH}_{3} \mathrm{OH}$ and its isotopes. These terms were found to have a significant contribution in fitting all the lines corresponding to $m=1$ state.

Rotational constants published earlier were used as initial guesses for calculating the torsional-rotational spectrum of these isotopes. The barrier height to internal rotation, $V_{2}$, was set to 0.839 and $1.906 \mathrm{MHz}$ for ${ }^{13} \mathrm{CC}_{5} \mathrm{H}_{6}-\mathrm{H}_{2} \mathrm{O}$ and $\mathrm{C}_{6} \mathrm{H}_{5} \mathrm{D}-\mathrm{H}_{2} \mathrm{O}$, respectively (being twice the experimental splitting observed for the $J=0 \rightarrow 1$ transition). The potential $V_{2}$ could not be determined from experimental splitting because of the dipole selection rule for these complexes which allows transitions within the same torsional manifold only.

The set of all transitions for the two isotopes are given in Table 3. The spectroscopic constants obtained from fitting transitions of $\mathrm{C}_{6} \mathrm{H}_{5} \mathrm{D}-\mathrm{H}_{2} \mathrm{O}$ and ${ }^{13} \mathrm{CC}_{5} \mathrm{H}_{6}-$ $\mathrm{H}_{2} \mathrm{O}$ are listed in Table 4. Centrifugal distortion terms determined from the fit are also given in Table 4. The $A$ rotational constant determined using $m=0$ and 1 transitions together is slightly different from that obtained using $m=0$ transitions alone. As the complex is a nearly prolate asymmetric top, the quantum number $K$ can be used to describe the energy levels. The program, we used (which is a modified ITRFIT code) however, denotes rotational energy levels associated with each torsional state $m$ by the label $J \tau m$ where $\tau$ gives the numbering of energy levels. Table 3 retains the $K$ 
labels for transitions following Eq. (1) for simplifying comparisons between the various isotopomers. The fits for ${ }^{13} \mathrm{CC}_{5} \mathrm{H}_{6}-\mathrm{H}_{2} \mathrm{O}$ and $\mathrm{C}_{6} \mathrm{H}_{5} \mathrm{D}-\mathrm{H}_{2} \mathrm{O}$ are qualitatively similar to that of the parent isotopomer for all $J^{\prime \prime} \rightarrow J^{\prime}=0 \rightarrow 1,1 \rightarrow 2$, and $2 \rightarrow 3$ transitions. However, for the $3 \rightarrow 4$ transitions the transition frequencies for $K=1$ and 2 could be fit for the parent symmetric top satisfactorily, but not so for the mono-substituted asymmetric tops. For both ${ }^{13} \mathrm{CC}_{5} \mathrm{H}_{6}-\mathrm{H}_{2} \mathrm{O}$ and $\mathrm{C}_{6} \mathrm{H}_{5} \mathrm{D}-\mathrm{H}_{2} \mathrm{O}$, instead of the two doublets, three lines which are approximately equally spaced have been observed. This could be due to some local perturbations of the corresponding energy levels that are not accounted for in this model. A closer inspection of Fig. 1 clearly highlights this possibility. The $K$ levels for the $m=1$ state are split for the symmetric $\mathrm{C}_{6} \mathrm{H}_{6}-\mathrm{H}_{2} \mathrm{O}$ and the slightly asymmetric ${ }^{13} \mathrm{CC}_{5} \mathrm{H}_{6}-\mathrm{H}_{2} \mathrm{O}$ and $\mathrm{C}_{6} \mathrm{H}_{5} \mathrm{D}-\mathrm{H}_{2} \mathrm{O}$ complexes due to the $L_{J a}$ term in the Hamiltonian (Eq. (2)). However, there is degeneracy in the energy levels of $\mathrm{C}_{6} \mathrm{H}_{6}-\mathrm{H}_{2} \mathrm{O}$, as the energy depends only on the product $\mathrm{Km}$. This degeneracy is lifted in the $m=1$ state for the slightly asymmetric ${ }^{13} \mathrm{CC}_{5} \mathrm{H}_{6}-\mathrm{H}_{2} \mathrm{O}$ and $\mathrm{C}_{6} \mathrm{H}_{5} \mathrm{D}-\mathrm{H}_{2} \mathrm{O}$ complexes, and these levels are split. This splitting is due to the asymmetry and the effective $V_{2}$ term introduced in the potential. It appears that for the $m K=+1$ and +2 levels, these two splittings become comparable leading to the observation of three instead of four transitions.

From the experimental spectra, it is clear that the splitting due to the asymmetry and the effective potential increases as $m K$ increases from $-J$ to $+J$. However, our model Hamiltonian does not show this. The energy levels for the $J=3$ and 4, and $m=1$ are shown in Fig. 2. Due to the scale used, the energy levels appear closer than they actually are. For clarity, the actual energies of all the states are given in Table 5, as well. As it is clear from Fig. 2 and Table 5, the model Hamiltonian leads to a splitting that dramatically decreases as $|m K|$ increases from 0 to $J$. Clearly, the effective potential should increase with $J^{2} \mathrm{Km}$. With that in mind, a term having the canonical form $P^{2}\left\{P_{a} P_{\gamma},(1-\cos 3 \gamma)\right\}$ was introduced in the Hamiltonian for fitting the spectra. With this, we tried to include $K=+1$ and +2 lines in the fit, but the rms deviation increased to several $\mathrm{MHz}$. It is likely that the perturbation is from some low frequency intermolecular modes that we have not considered. These energy levels start at $15 \mathrm{~cm}^{-1}$. Experimental observations of low frequency intermolecular vibrational modes may help in resolving all the complexities of the rotational spectra of this important class of dimers.

\section{Conclusion}

The recently derived vibrational-rotational-torsional Hamiltonian by Duan et al. has been used to identify and fit the $m=1$ lines of the symmetric $\mathrm{C}_{6} \mathrm{H}_{6}-\mathrm{H}_{2} \mathrm{O}$ complex and its mono-substituted asymmetric isotopomers. With this approach, the $m=1$ spectrum of the monosubstituted asymmetric dimers could be fitted as successfully as that of the parent symmetric dimer. In addition, previous assignments of $m=0$ lines of ${ }^{13} \mathrm{CC}_{5} \mathrm{H}_{6}-\mathrm{H}_{2} \mathrm{O}$ and ${ }^{13} \mathrm{CC}_{5} \mathrm{H}_{6}-\mathrm{D}_{2} \mathrm{O}$ have been corrected. This has resulted in the determination of the $C$ rotational constant for the ${ }^{13} \mathrm{CC}_{5} \mathrm{H}_{6}$, which is effectively the same as the $A$ rotational constant of the complex. Rotational and

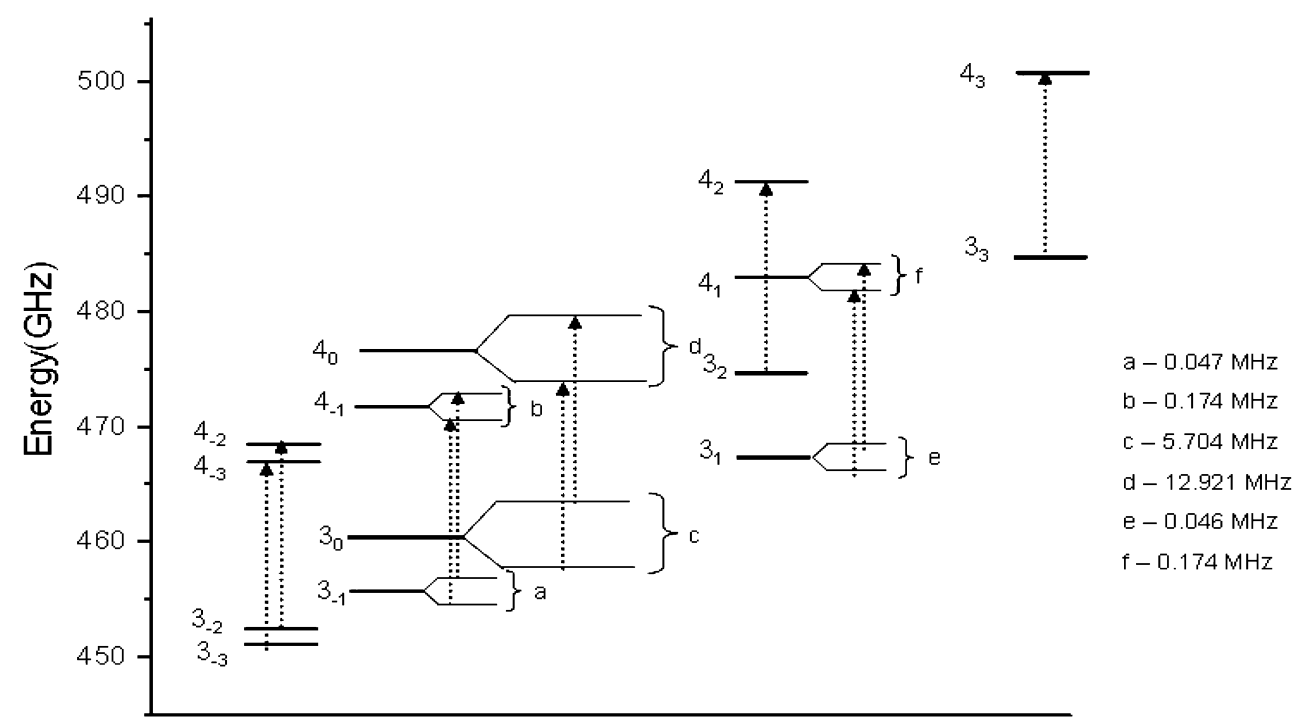

Fig. 2. Energy level diagram for the $m=1$ levels of ${ }^{13} \mathrm{CC}_{5} \mathrm{H}_{6}-\mathrm{H}_{2} \mathrm{O}$. The model Hamiltonian shows a splitting in $|K|$ levels that decrease as $|K|$ increases from 0 to $J$ and the experiment shows that, this splitting increases as $K$ increases from $-J$ to $+J$. According to the experimental spectrum observed, the splitting of the $K$ due to the effective potential, $V_{2}$, and asymmetry becomes comparable to the splitting in energy level. Due to the scale used, the energy levels appear closer than they actually are. See Table 5 for the calculated energies. 
Table 5

Energies (in MHz) of the $m=1, J=3$ and 4 plotted in Fig. 2

\begin{tabular}{|c|c|c|c|c|c|c|c|c|c|c|}
\hline$K$ & $J$ & $\tau$ & $m$ & & $\Delta E$ & $K$ & $J$ & $\tau$ & $m$ & \\
\hline \multirow[t]{2}{*}{$\overline{-3}$} & 3 & 1 & 1 & 451425.370 & 15720.574 & -3 & 4 & 3 & 1 & 467145.944 \\
\hline & 3 & 2 & 1 & 451425.370 & 15720.574 & & 4 & 4 & 1 & 467145.944 \\
\hline \multirow[t]{2}{*}{-2} & 3 & 3 & 1 & 453011.592 & 15742.036 & -2 & 4 & 5 & 1 & 468753.628 \\
\hline & 3 & 4 & 1 & 453011.592 & 15742.038 & & 4 & 6 & 1 & 468753.630 \\
\hline \multirow[t]{2}{*}{-1} & 3 & 5 & 1 & 456199.831 & 15758.513 & -1 & 4 & 7 & 1 & 471958.344 \\
\hline & 3 & 6 & 1 & 456199.878 & 15758.640 & & 4 & 8 & 1 & 471958.518 \\
\hline \multirow[t]{2}{*}{0} & 3 & 7 & 1 & 460988.572 & 15771.189 & 0 & 4 & 9 & 1 & 476759.760 \\
\hline & 3 & 8 & 1 & 460994.276 & 15778.405 & & 4 & 10 & 1 & 476772.681 \\
\hline \multirow[t]{2}{*}{1} & 3 & 9 & 1 & 467387.947 & 15793.280 & 1 & 4 & 11 & 1 & 483181.227 \\
\hline & 3 & 10 & 1 & 467387.993 & 15793.408 & & 4 & 12 & 1 & 483181.401 \\
\hline \multirow[t]{2}{*}{2} & 3 & 11 & 1 & 475391.118 & 15817.072 & 2 & 4 & 13 & 1 & 491208.190 \\
\hline & 3 & 12 & 1 & 475391.118 & 15817.073 & & 4 & 14 & 1 & 491208.191 \\
\hline \multirow[t]{2}{*}{3} & 3 & 13 & 1 & 485002.491 & 15848.863 & 3 & 4 & 15 & 1 & 500851.354 \\
\hline & 3 & 14 & 1 & 485002.491 & 15848.864 & & 4 & 16 & 1 & 500851.355 \\
\hline
\end{tabular}

$\Delta E$ is the transition frequencies predicted for $J=3 \rightarrow 4, m=1$.

centrifugal distortion constants for the $m=0$ and 1 states for the complexes have been reported.

\section{Acknowledgments}

One of us (E.A.) wishes to express his gratitude to the Late Professor H.S. Gutowsky in whose laboratory the measurements were made and his former colleague Dr. T.I. Emilsson for help. Authors thank Dr. Amine-Taleb Bendiab for providing the ITRFIT code he had developed. Sagarika Dev helped during the earlier stages of this work. E.A. and M.S.K. wish to thank the Department of Science and Technology, Government of India for financial support through project grants. E.A. thanks Director, I.I.Sc. and Council of Scientific and Industrial Research for partial support. M.S.K. acknowledges support by the Computer Centre, IIT Madras.

\section{References}

[1] M. Levitt, M.F. Perutz, J. Mol. Biol. 201 (1998) 178.

[2] M.F. Perutz, in: A. Zewail (Ed.), The Chemical Bond, Academic Press, New York, 1992, pp. 17-30.

[3] S.K. Burley, G.A. Petsko, FEBS Lett. 203 (1986) 139.

[4] J.D. Augspurger, C.E. Dykstra, T.S. Zwier, J. Phys. Chem. 96 (1992) 7252.

[5] S. Suzuki, P.G. Green, R.E. Bumgarner, S. Dasgupta, W.A. Goddard III, G.A. Blake, Science 257 (1992) 942.

[6] H.S. Gutowsky, T. Emilsson, E. Arunan, J. Chem. Phys. 99 (1993) 4883.
[7] G.T. Fraser, F.J. Lovas, R.D. Suenram, D.D. Nelson Jr., W. Klemperer, J. Chem. Phys. 84 (1986) 5983.

[8] W. Gordy, R.L. Cook, Microwave Molecular Spectra, Wiley, New York, 1984.

[9] E. Arunan, T. Emilsson, H.S. Gutowsky, J. Chem. Phys. 101 (1994) 861.

[10] T. Emilsson, H.S. Gutowsky, G. de Oliveira, C.E. Dykstra, J. Chem. Phys. 112 (2000) 1287.

[11] A. Taleb-Bendiab, K.W. Hilling II, R.L. Kuczkowski, J. Chem. Phys. 97 (1992) 2996.

[12] F. Rohart, J. Mol. Spectrosc. 57 (1975) 301.

[13] A.P. Cox, J. Mol. Struct. 97 (1983) 61.

[14] D. Kivelson, E.B. Wilson Jr., J. Chem. Phys. 20 (1952) 1575.

[15] A.C. Legon, P.D. Aldrich, W.H. Flygare, J. Chem. Phys. 75 (1981) 625 .

[16] P.R.R. Langridge-Smith, A.P. Cox, J. Chem. Soc. Faraday Trans. 279 (1982) 1089.

[17] Y.B. Duan, H.M. Zhang, K. Takagi, J. Chem. Phys. 104 (1996) 3914.

[18] Y.B. Duan, L. Wang, K. Takagi, J. Mol. Spectrosc. 193 (1999) 418.

[19] M.R. Aliev, J.K.G. Watson, in: K. Narahari Rao (Ed.), Molecular Spectroscopy: Modern Research, vol. III, Academic Press, New York, 1985, p. 1.

[20] E. Arunan, S. Dev, P.K. Mandal, App. Spectrosc. Rev. 39 (2004) 131.

[21] H. Kollenstein, S. Piccirillo, M. Quack, M. Snels, Mol. Phys. 71 (1990) 759.

[22] R.J.J.M. Steenvoorden, M.H. Vasconcelos, P.G. Kistemaker, T.L. Wedding, J. Mol. Spectrosc. 161 (1993) 17.

[23] M. Oldani, T.K. Ha, A. Bauder, Chem. Phys. Lett. 115 (1985) 317.

[24] C. Richard Quade, R.D. Suenram, J. Chem. Phys. 73 (1980) 1127.

[25] J.K.G. Watson, in: J.R. Durig (Ed.), Vibrational Spectra and Structure, vol. 6, Elsevier, Amsterdam, 1977, Chapter 1. 